\title{
Treatment with Olanzapine is Associated with Modulation of the Default Mode Network in Patients with Schizophrenia
}

\author{
Fabio Sambataro',3, Giuseppe Blasi', Leonardo Fazio', Grazia Caforio', Paolo Taurisano', \\ Raffaella Romano', Annabella Di Giorgio', Barbara Gelao', Luciana Lo Bianco', Apostolos Papazacharias', \\ Teresa Popolizio², Marcello Nardini' ${ }^{1}$ and Alessandro Bertolino*, I,2 \\ 'Psychiatric Neuroscience Group, Department of Neurological and Psychiatric Sciences, University of Bari, Bari, Italy; ${ }^{2}$ Department of \\ Neuroradiology, IRCCS 'Casa Sollievo della Sofferenza', San Giovanni Rotondo, FG, Italy; ${ }^{3}$ Clinical Brain Disorders Branch, Genes, Cognition and \\ Psychosis, NIMH, NIH, Bethesda, MD, USA
}

Earlier studies have shown widespread alterations of functional connectivity of various brain networks in schizophrenia, including the default mode network (DMN). The DMN has also an important role in the performance of cognitive tasks. Furthermore, treatment with second-generation antipsychotic drugs may ameliorate to some degree working memory (WM) deficits and related brain activity. The aim of this study was to evaluate the effects of treatment with olanzapine monotherapy on functional connectivity among brain regions of the DMN during WM. Seventeen patients underwent an 8-week prospective study and completed two functional magnetic resonance imaging ( $(\mathrm{MRI})$ scans at 4 and 8 weeks of treatment during the performance of the N-back WM task. To control for potential repetition effects, 19 healthy controls also underwent two $\mathrm{PMRI}$ scans at a similar time interval. We used spatial group-independent component analysis (ICA) to analyze fMRI data. Relative to controls, patients with schizophrenia had reduced connectivity strength within the DMN in posterior cingulate, whereas it was greater in precuneus and inferior parietal lobule. Treatment with olanzapine was associated with increases in DMN connectivity with ventromedial prefrontal cortex, but not in posterior regions of DMN. These results suggest that treatment with olanzapine is associated with the modulation of DMN connectivity in schizophrenia. In addition, our findings suggest critical functional differences in the regions of DMN.

Neuropsychopharmacology (2010) 35, 904-912; doi:I0.1038/npp.2009.192; published online 2 December 2009

Keywords: default mode network; schizophrenia; antipsychotics; working memory; ventromedial prefrontal cortex; $\mathrm{PMRI}$

\section{INTRODUCTION}

Alterations of functional connectivity of the brain have been implicated in the pathophysiology of schizophrenia (Friston and Frith, 1995; Meyer-Lindenberg et al, 2001; Weinberger et al, 1992). Although initial studies focused on abnormal dynamic interactions among functionally segregated brain regions co-activated during a task (Meyer-Lindenberg et al, 2001), a growing number of reports has recently suggested that disruptions in the default mode network (DMN) may have a role in the cognitive deficits found in schizophrenia (see (Broyd et al, 2009) for a review).

The DMN comprises a set of brain regions whose activity increases during rest and is attenuated during an active task (Mazoyer et al, 2001; Raichle et al, 2001). This brain

* Correspondence: Professor A Bertolino, Dipartimento di Scienze Neurologiche e Psichiatriche, Università degli Studi di Bari, Piazza Giulio Cesare, Bari 9 - 70124, Italy, Tel: + 39080 5478572, Fax: + 39080 5593204, E-mail: a.bertolino@psichiat.uniba.it

Received 21 July 2009; revised 7 October 2009; accepted 26 October 2009 network includes midline areas such as the ventromedial prefrontal cortex (vmPFC), the posterior cingulate cortex (pCC), the precuneus, as well as bilateral inferior parietal lobule (IPL) (Raichle et al, 2001). Activity in the DMN has been associated with stimulus-unrelated thoughts, lapses of attention and mind wandering (Mason et al, 2007). Different roles have been hypothesized for this network ranging from monitoring external environment ('watchfulness'), to internal mentation, for example, autobiographical memory, theory of mind, moral decision making and prospective thoughts (Gusnard et al, 2001). Interestingly, activity of the DMN shows a strong negative correlation, 'anticorrelation', with activity in task-related networks (Fox et al, 2005). This finding suggests the existence of two different modes of information processing: one with predominant DMN activity, which is relatively unfocused on external objects and characterized by mental exploration, whereas the other is focused on active processing of external stimuli (see (Buckner et al, 2008) for a review). Importantly, integrity of the DMN is also important for allocation of attentional resources needed for cognitive processing and has been associated with working memory 
(WM) performance (Hampson et al, 2006; Sambataro et al, 2008).

Although brain functions proposed for the DMN are impaired in patients with schizophrenia (Buckner et al, 2008), neuroimaging studies have reported inconsistent findings in the DMN of these individuals. Increased (Garrity et al, 2007; Harrison et al, 2007; Whitfield-Gabrieli et al, 2009; Zhou et al, 2007) or decreased (Liang et al, 2006; Pomarol-Clotet et al, 2008) activity and connectivity have been reported in the DMN network at the level of individual regions as well as of the global network. Different issues may have contributed to lack of consistent findings, including the method used for estimation of connectivity (Murphy et al, 2009). For example, the use of global signal regression, a standard preprocessing step of univariate seed-based connectivity analyses, has been questioned for the study of $\mathrm{DMN}$ as having a potential biasing effect in the estimation of the magnitude of the anticorrelations. Given that the use of this technique is still controversial (Fox et al, 2009; Murphy et al, 2009; Weissenbacher et al, 2009), other statistical approaches that do not rely on this preprocessing method such as independent component analysis (ICA) may yield more reliable results.

Another crucial determinant potentially impacting data consistency and DMN activity may be the effect of pharmacological treatment with antipsychotics. Although some reports are questioning the size of the effect and specificity of the cognitive effects of second-generation antipsychotics (Goldberg et al, 2007; Keefe et al, 2007), some evidence shows that this treatment is associated with improvement of some of the cognitive deficits of schizophrenia including WM (Bertolino et al, 2004; Purdon et al, 2000). Furthermore, second-generation antipsychotic treatment appears to modulate brain physiology underlying WM function with reduced activation of dorsolateral prefrontal cortex (DLPFC) and parietal cortex for better levels of performance, suggesting increased neural efficiency (Bertolino et al, 2004). Moreover, other studies have indicated that systemic administration of levodopa and apomorphine in healthy humans or in patients with Parkinson's disease can modify the activity of the DMN (Argyelan et al, 2008; Nagano-Saito et al, 2009) further suggesting the potential relationship between dopamine signaling and DMN modulation. On the other hand, despite these earlier studies, the effect of treatment with antipsychotics on the DMN is still unknown.

In this study, we hypothesized that the effects of treatment with olanzapine, a second-generation antipsychotic drug, on WM brain physiology and behavioral performance can also be associated with modulation of connectivity within the DMN. Thus, we performed a longitudinal study in previously untreated patients with schizophrenia undergoing 8 weeks of treatment with olanzapine monotherapy. Patients were studied using spatial group ICA with fMRI during WM at $4\left(t_{1}\right)$ and 8 weeks of treatment $\left(t_{2}\right)$. To control for potential well-known effects of task practice and learning (Goldberg et al, 2007), we also scanned normal controls twice $\left(t_{1}, t_{2}\right)$ with a time interval similar to that of patients.

\section{MATERIALS AND METHODS}

\section{Subjects}

A total of 19 patients with schizophrenia and 19 healthy controls, matched for age, gender, race, handedness participated (Oldfield, 1971) and socioeconomic status (Hollingshead and Redlich, 1958) participated in this study (Table 1). All subjects underwent clinical assessment following the Structured Clinical Interview for DSM-IV Axis I Disorders (SCID-IV; First et al, 1996). Exclusion criteria included past history or presence of any medical, neurological disorders, drug or alcohol abuse, active drug use in the past year, past head trauma with loss of consciousness. Normal controls were excluded if presented with past history or current diagnosis of psychiatric disorders as documented by SCID-IV.

At the time of enrollment, all patients were experiencing acute exacerbation of psychosis requiring hospitalization. Nine patients were diagnosed with schizophreniform disorder and all of them were diagnosed with schizophrenia at a later follow-up. In all 13 patients were drug-naive and 6 had been drug-free for at least 2 weeks from oral antipsychotics or from at least two cycles of depot antipsychotic treatment. Clinical symptoms in patients were assessed on the day of study entry, at 4 weeks $\left(t_{1}\right)$, and at 8 weeks $\left(t_{2}\right)$ by a trained psychiatrist using the Positive and Negative Syndrome Scale (PANSS; see Table 2 for clinical data). All patients were treated with oral olanzapine monotherapy. Titration was allowed for the first 2 weeks, and then the dose was kept constant throughout the remaining 6 weeks of treatment (mean dose $20 \mathrm{mg} /$ day; $\mathrm{SD}=6.9 \mathrm{mg} /$ day). Given the lack of reliable algorithms to handle missing data points in statistical imaging, only subjects with two longitudinal fMRI scans were selected for this study.

All participants gave written informed consent, approved by the Institutional Review Board of the University of Bari, Bari (Italy), to take part in the experiment.

\section{Experimental Task}

All subjects performed the N-back WM task as described elsewhere (Callicott et al, 1999). Briefly, subjects saw numbers (1-4) shown in pseudorandom sequence and displayed at the corners of a diamond-shaped box. A nonmemory guided control condition ( 0 -back) that required subjects to identify the number currently seen, alternated with the 2-back WM condition, in which subjects were asked to recall the number seen two stimuli before, while continuing to encode additionally incoming stimuli.

Table I Demographics of the Sample

\begin{tabular}{|c|c|c|c|}
\hline & Patients & Normal controls & Difference \\
\hline$N$ & 19 & 19 & \\
\hline Male/Female ratio & $15: 4$ & $15: 4$ & NS \\
\hline Age ( $M \pm S D$, years) & $24.8 \pm 5.7$ & $26.3 \pm 6.9$ & $p=0.47$ \\
\hline Handedness & $0.80 \pm 0.4$ & $0.64 \pm 0.4$ & $p=0.22$ \\
\hline 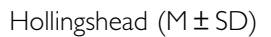 & $28.8 \pm 15.6$ & $32.8 \pm 16.0$ & $p=0.43$ \\
\hline
\end{tabular}


Table 2 Clinical Data

\begin{tabular}{|c|c|c|}
\hline & $\mathbf{M}$ & SD \\
\hline Drug free (months), $N=6$ & 11.2 & 13.2 \\
\hline \multicolumn{3}{|c|}{ Drug-naive, $N=13,9$ of whom First-Episode } \\
\hline Age of onset & 21.4 & 4.2 \\
\hline Length of illness (months) & 44.1 & 67 \\
\hline Mean dose olanzapine (mg/day) & 20 & 6.9 \\
\hline \multicolumn{3}{|l|}{ PANSS baseline } \\
\hline PANSS total & 101.6 & 25.2 \\
\hline PANSS positive & 25.6 & 6.0 \\
\hline PANSS negative & 23.9 & 10.7 \\
\hline PANSS general & 52.2 & 13.7 \\
\hline \multicolumn{3}{|l|}{ PANSS 4 weeks } \\
\hline PANSS total & 68.47 & 18.88* \\
\hline PANSS positive & 16.95 & $4.61^{*}$ \\
\hline PANSS negative & 17.89 & $6.87^{*}$ \\
\hline PANSS general & 35.26 & 8.90* \\
\hline \multicolumn{3}{|l|}{ PANSS 8 weeks } \\
\hline PANSS total & 60.63 & 15.66* \\
\hline PANSS positive & 14.16 & $4.65^{*}, \dagger$ \\
\hline PANSS negative & 15.58 & $7.05^{*}, \dagger$ \\
\hline PANSS general & 30.37 & $7.48^{*}+\dagger$ \\
\hline
\end{tabular}

Abbreviations: M, Mean; PANNS, Positive and Negative Syndrome Scale. * $p<0.005$ from baseline; ${ }^{\dagger} p<0.005$ from 4 weeks.

Performance was recorded through a fiber optic response box which allowed measurement of behavioral data as the number of correct responses (accuracy) and reaction time (RT). Data recordings of two patients were lost because of a computer glitch. The stimuli were arranged in a blockdesign, consisting of eight 30-s blocks: four blocks of the control condition alternating with four blocks of the 2-back WM condition.

\section{Image Acquisition}

Blood-oxygen level dependent fMRI was performed on a GE Signa (Milwaukee, WI) 3T scanner at $t_{1}$ and $t_{2}$. A gradient echo Blood-oxygen level dependent-EPI pulse sequence was used to acquire 120 images per run. Each functional image consisted of $206-\mathrm{mm}$-thick axial slices covering the entire cerebrum and most of the cerebellum $(T R=2000 \mathrm{~ms}$; $\mathrm{TE}=30 \mathrm{~ms}$; field of view $=24 \mathrm{~cm}$; flip angle $=90^{\circ}$ ).

\section{Data Analysis}

Demographics, behavioral, and clinical data. Two sample $t$-tests and repeated measures ANOVAs were used to compare demographics and behavioral data, respectively. Chi-square analyses were performed to compare categorical variables. Clinical data were compared using paired $t$-tests.
Image analysis.

Preprocessing: Data were pre-processed and analyzed using Statistical Parametrical Mapping 2 (SPM; http:// www.fil.ion.ucl.ac.uk). The first four volumes were discarded to allow for T1 equilibration effects. All functional volumes were realigned to the first volume acquired and spatially normalized to the Montreal Neurological Institute (MNI) standard brain in the space of Talairach and Tournoux (1988). Smoothing was carried out with a 10$\mathrm{mm}$ full width half maximum isotropic 3D Gaussian kernel.

Independent component analysis: One group of spatial ICA was performed on preprocessed data using the Group ICA of fMRI Toolbox [GIFT; http://icatb.sourceforge.net, (Calhoun et al, 2001)] entering the data of all subjects for both time points. The dimensionality of functional data for each subject was reduced using three consecutive steps of Principal Component Analysis alternating with data concatenation across the subjects, resulting in one aggregate mixing matrix for all subjects. An ICA decomposition using the Infomax algorithm was used to extract 21 independent components (IC), consisting of group spatial maps and the related time courses (TC) of the estimated signal. Individual participant's spatial maps for each IC, which are the voxelwise IC loadings, represent local strength of functional connectivity and reflect the correspondence between the estimated TC in each voxel and the average TC of the network itself. Given that in spatial ICA, the individual subject TC is assumed to be constant across the entire brain, the voxel-wise estimated signal deviations from the average network TC reflects local changes in the strength of functional connectivity within a given network. The minimum description length criteria were used to estimate the order selection that is the number of ICs from the smoothed data sets after taking into account the spatial and temporal correlation of the fMRI data (Li et al, 2007). These components were then used to back-reconstruct to individual ICs using the aggregate mixing matrix created during the reduction steps of dimensionality data. Each voxel value of the IC map for each participant was calibrated using $z$-scores for between participant comparisons.

Eventually, two components that resulted unstable as suggested by a coefficient of stability (Iq) lower than 0.95 calculated by 50 bootstrapped permutated estimations of the ICs (ICASSO; Himberg et al, 2004) or showed artifactual patterns defined by those ICs with a spatial correlation greater than $R^{2}=0.02$ with white matter and $R^{2}=0.05$ for CSF (Kim et al, 2009) were removed from the analysis. The ICs that had (1) the highest negative Pearson's $r$ correlation between its TC and the 2-back condition (a box-car function convolved with a double-Gaussian hemodynamic response function) and (2) the highest spatial correlation with a DMN template provided with the ICA toolbox were selected for further analyses.

Spatial analyses: Each subject's DMN-IC was entered into SPM5 and analyzed using second level random-effects analyses. Repeated measures ANCOVA with the normalized 2-back performance (ratio between 2-back and 0-back accuracy) as a covariate of no interest and time as a within subjects variable was used to compare DMN-IC spatial 
maps across diagnostic groups at each time point. Additional planned comparisons were performed to assess differences across diagnostic groups at each time point. To examine differences in positive and negative spatial maps separately, we created a positive and negative mask, respectively, using a one-sample $t$-test across all subjects. Average connectivity strength was extracted in significant clusters and correlated with WM performance computed as a ratio between percent accuracy at 2-back and at 0 -back.

A statistical threshold of $p<0.05$ with False-DiscoveryRate (FDR; Genovese et al, 2002) small-volume-correction with $\mathrm{q}-\mathrm{FDR}=0.05$ was used to identify significant differences within regions of interest. Regions of interest were created using WFU pickatlas software version 1.04 (Functional MRI Laboratory at the Wake Forest University School of Medicine, http://www.rad.wfubmc.edu/fmri) and comprised the following a priori regions as part of the standard default mode: vmPFC, anterior cingulate cortex (BA24/32), and the IPL, as well as the posterior cingulate, and precuneus (Raichle et al, 2001). All coordinates are reported in MNI system.

Stimulus-related response: Averaged IC responses to the WM task were calculated for the DMN-IC TC for patients and normal controls separately during the 2-back condition using the deconvolution algorithm by Eichele et al (2008). Stimulus-related response depicts the extent of the modulation of the DMN TC during presentation of a WM stimulus of null duration. Averaged TCs were overlaid for both groups for $17 \mathrm{~s}$ following the stimulus onset to estimate the full extent of the TC response to stimuli.

\section{RESULTS}

\section{Behavioral Performance}

There was a significant effect of diagnosis $[\mathrm{F}(1,34)=13.596$, $p=0.0007]$ on task accuracy: patients with schizophrenia had reduced performance relative to normal controls. There was also an effect of time $[\mathrm{F}(1,34)=9.151, p=0.004]$ indicating that subjects at $t_{1}$ had lower performance relative to $t_{2}$. There was no interaction of diagnosis by time of observation $(p>0.1)$. Even though both groups of subjects tended to improve accuracy over time, a $t$-test for dependent samples demonstrated that the difference was significant in patients $\left(t_{16}=2.05, p=0.05\right)$ but not in controls $\left(t_{18}=1.61, p=0.12\right)$, suggesting an effect of olanzapine on WM accuracy in patients. There was a significant effect of diagnosis $[\mathrm{F}(1,34)=5.1299, p=0.03]$ on reaction time, with patients with schizophrenia being slower relative to normal controls. There was no effect of time of observation $(p>0.1)$ or interaction of diagnosis by time of observation $(p>0.1)$ on reaction time.

\section{Imaging Results}

Default mode network component. One IC spanning across the DMN regions and anticorrelated with the task was identified for all subjects across both time points (Figure 1a).
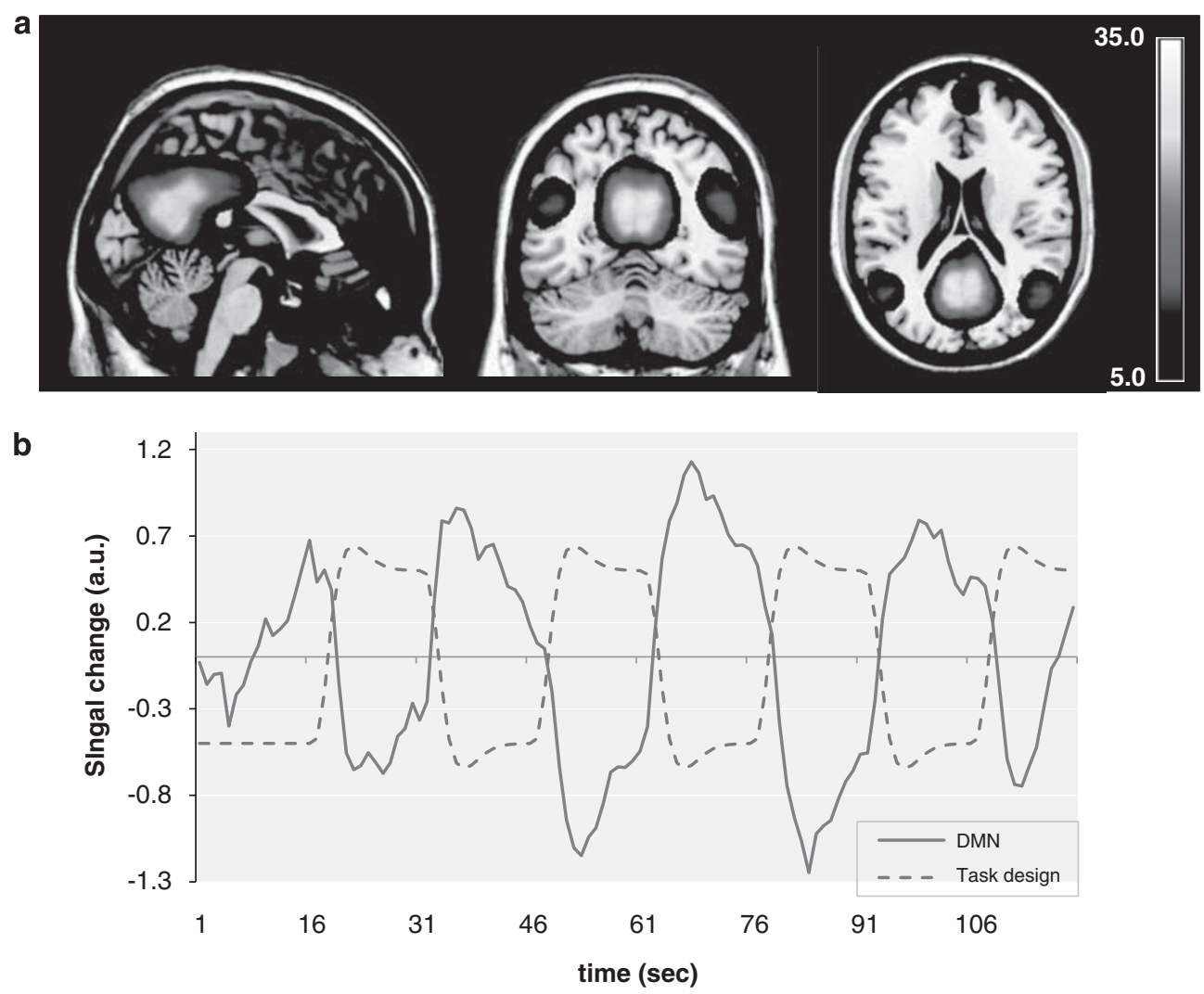

Figure I Default mode network (DMN). One DMN-IC comprising spatial maps and time courses was identified across both time points for all the subjects. (a) Axial maps display the spatial pattern of the independent components identified. (b) Time courses represent the temporal profile of DMN each component (blue continuous line) overlaid on the paradigm 'box-car' design (red dashed line). All images are thresholded at $p=0.05$ corrected for multiple comparisons. Color bar indicates $t$-scores. 
This IC included midline regions vmPFC, pCC, precuneus (BA5/7), as well as bilateral IPL. This IC had high spatial correlation with a DMN template, $R^{2}=0.33$ and high temporal anticorrelation with the timing of the 2-back condition of task, $r=-0.65$ (Figure $1 \mathrm{~b}$ ).

\section{Between group comparisons.}

Spatial extent: Effect of Diagnosis Patients with schizophrenia had increased connectivity with the DMN in left IPL (BA39, $x=-56, y=-67, z=23, Z=4.03, p=0.00003$ FDR corrected) as well as in precuneus (BA7, $x=4, y=-79$, $z=45, Z=3.33, p=0.0004$ with $\mathrm{q}-\mathrm{FDR}=0.09$ ). In addition, patients had decreased connectivity with the DMN in pCC (BA23/31, $x=4, y=-52, z=26, Z=3.34, p=0.0004$ FDR corrected; Figure 2).

Effect of time There was no significant effect of time.

Time by diagnosis There was a time by diagnosis interaction in vmPFC (BA10, $x=0, y=49, z=-7$, $Z=3.02, p=0.001$ FDR corrected; Figure 3a). In patients with schizophrenia the strength of connectivity increased at $t_{2}$ relative to $t_{1}$ with respect to normal controls (Figure $3 \mathrm{~b}$ ). Post hoc analysis revealed that patients with schizophrenia had significantly greater connectivity strength extracted in the vmPFC cluster at $t_{2}$ relative to $t_{1}(p=0.01)$. However, this was not true in normal controls $(p>0.1)$. In addition, at $t_{2}$ connectivity strength in patients in this region was greater relative to normal controls $(p=0.01)$. Strength of connectivity in vmPFC was positively correlated with WM accuracy in patients, but not in normal controls (controls: $t_{1}: r=-0.20, p>0.1 ; t_{2}: r=-0.15, p>0.1 ;$ Figure $\left.3 c\right)$.
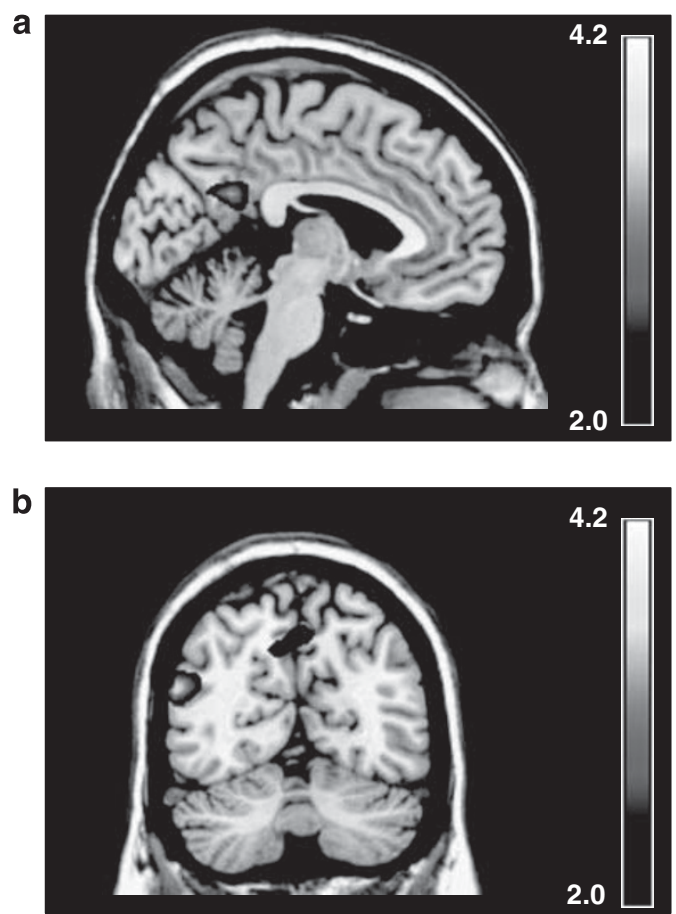

Figure 2 Effect of diagnosis on brain connectivity in the DMN. Patients with schizophrenia show decreased connectivity strength within the DMN in PCC (a) as well as increased connectivity in left-Inferior Parietal Lobule (BA39) and precuneus (BA7) relative to normal controls (b). Statistical t-map of DMN connectivity overlaid on the MNI brain template. Color bar indicates $t$-scores.
Furthermore, in patients the correlation was significant at $t_{1}$ but not at $t_{2}$ (patients: $t_{1}: r=0.49, p=0.05 ; t_{2} ; r=0.16, p>0.1$ ).

Average stimulus-related responses. Average stimulus responses of DMN IC to the WM condition had a smaller negative modulation of this signal in patients relative to normal controls. This difference in WM-related responses decreased at $t_{2}$ relative $t_{1}$ (Figure 4 ).

\section{Negative DMN component}

The negative component of DMN had a pattern similar to the typical WM task described during 2-back task (Supplementary Figure S1). This network of brain regions included bilaterally dorsolateral (DLPFC) and ventrolateral prefrontal cortex, frontal pole, caudate, anterior cingulate, and lateral posterior parietal cortex. This network demonstrated main effects and interaction between diagnosis and treatment in the region of DLPFC previously shown to be responsive to treatment with olanzapine as in earlier studies (Bertolino et al, 2004; see Supplementary Materials for further details). In normal controls $\left(t_{1}: r=0.40, p=0.09\right.$; $\left.t_{2}=0.39, p=0.1\right)$ but not patients $\left(t_{1}: r=-0.03, p>0.1\right.$; $\left.t_{2}=-0.14, p>0.1\right)$ there was a statistical trend for correlation between DLPFC connectivity strength and WM accuracy.

\section{DISCUSSION}

In this study we investigated the effect of olanzapine on DMN connectivity in patients with schizophrenia. We found that patients have greater strength of connectivity in the IPL and precuneus as well as reduced connectivity in pCC. These effects were not significantly affected by treatment with olanzapine. On the other hand, our data indicate an interaction of time by diagnosis on connectivity strength in vmPFC where patients with schizophrenia had greater connectivity strength with the rest of DMN during olanzapine treatment. In patients with schizophrenia the extent of DMN connectivity strength in vmPFC positively correlated with WM accuracy at $t_{1}$ but not at $t_{2}$.

Previous imaging studies have reported widespread alterations of DMN in patients with schizophrenia (see Broyd et al, 2009 for a review). Our findings indicate decreased functional connectivity with the DMN in pCC as well as increased connectivity strength in parietal cortex, including precuneus and IPL, in patients with schizophrenia. pCC is an important node of the DMN and a crucial cortical hub for interconnecting segregated brain areas, that is, anterior and posterior midline systems that are $\mathrm{mPFC}$ and entorhinal cortex (Greicius et al, 2009). As a result of these connections pCC may have an important role in cognitive functions including episodic memory (Greicius et al, 2003) and WM (Hampson et al, 2006; Sambataro et al, 2008). In addition, disruption of functional coupling with pCC is associated with ADHD (Castellanos et al, 2008) and cognitive decline in physiological (Sambataro et al, 2008) and pathological aging (Greicius et al, 2004). The relative lack of disease specificity of these findings suggests that this mechanism reflects basic disruption of the brain systems associated with memory dysfunction across different 

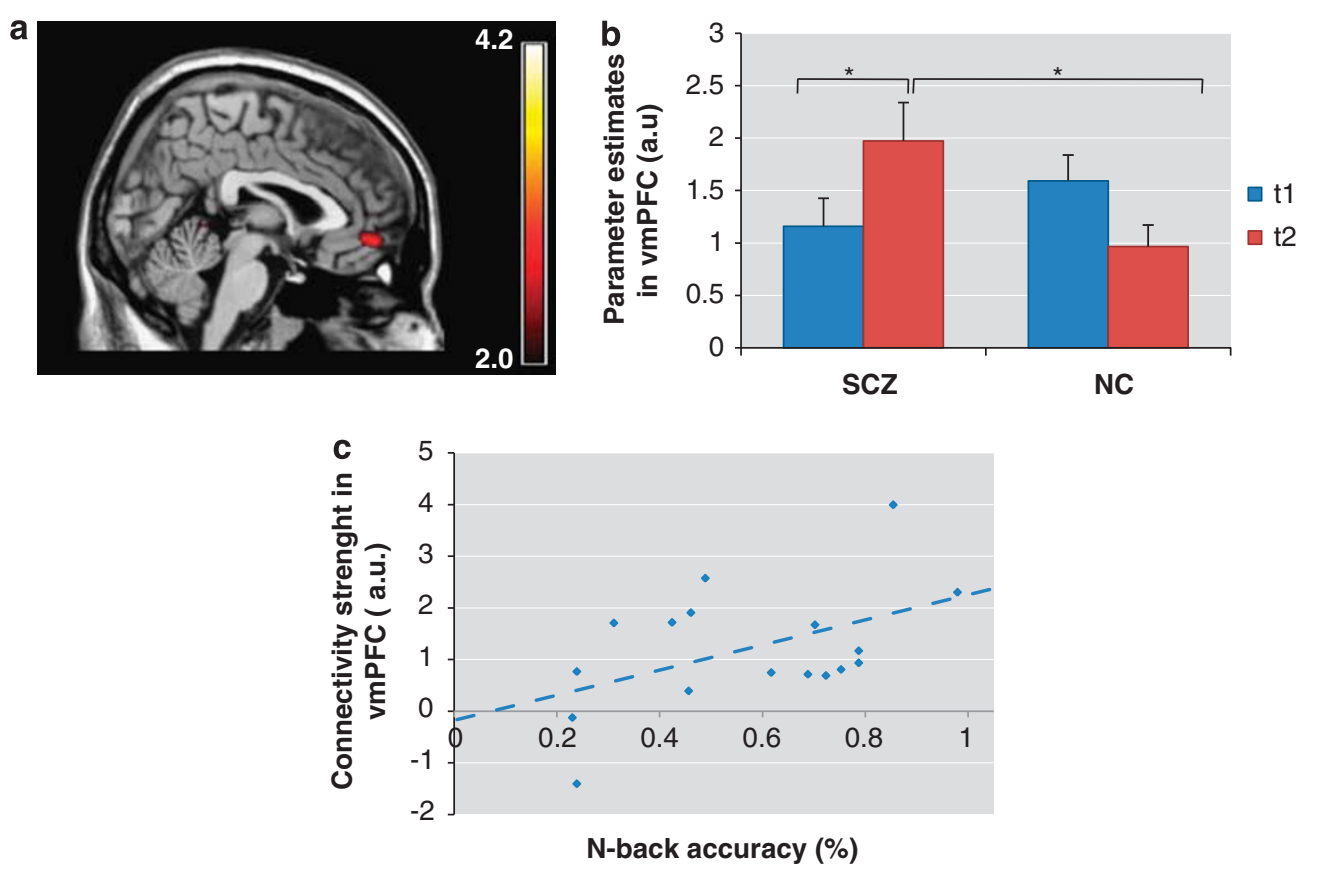

Figure 3 Time by diagnosis interaction on brain connectivity in the DMN. In patients with schizophrenia (SCZ) connectivity strength within the DMN increased in ventromedial prefrontal cortex (VmPFC) (a); at $t_{2}$ (red) relative to $t_{1}$ (blue) when compared with normal controls (NC) (b); connectivity strength in VMPFC was positively correlated with task performance in patients at $t_{1}(r=0.45, p=0.04)$ (c); statistical t-maps of DMN connectivity are overlaid on the MNI brain template. Color bar indicates $t$-scores. Bar plots report parameter estimates of the time by diagnosis contrast from the ANCOVA extracted from the vmPFC cluster and measured in arbitrary units (a.u.). Dashed lines indicate the trendline. *P=0.0 I.

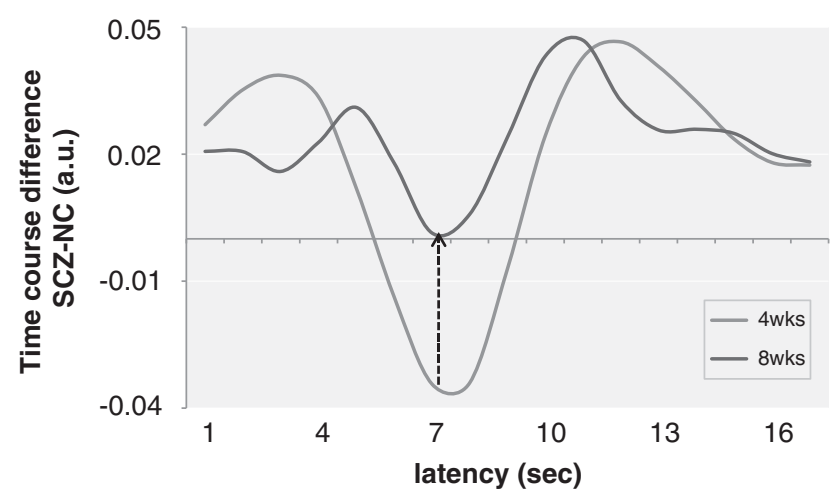

Figure 4 Time by diagnosis interaction on stimulus-related responses. At $t_{1}$ patients had a smaller stimulus-related decrease of the time course amplitude relative to normal controls, and this difference decreased at $t_{2}$ $Y$ axis indicates the difference (patients - normal controls, SCZ-NC) of time course amplitude of the DMN-IC patients with schizophrenia relative to normal controls in arbitrary units (a.u.) as calculated by deconvolving the estimated signal for the 2-back condition, $X$ axis indicates latency from the working memory stimulus onset expressed in seconds. The dashed arrow indicates the effect of time on the group differences.

categorical diagnostic conditions. Thus, decreased connectivity strength between pCC and the other regions of DMN may contribute to the WM deficits observed in patients with schizophrenia. On the other hand, connectivity of the parietal regions, that is, precuneus and IPL, with the DMN has crucial anatomical and functional differences within posterior DMN regions. Cytoarchitectonically, the precuneus is a fully differentiated isocortex as opposed to the limbic cortex in pCC, and it also has structural connections with different thalamic nuclei and with occipital, parietal, and frontal areas (Buckwalter et al, 2008). This region is associated with visual processing, motor planning (Cavada and Goldman-Rakic, 1989), cognitive functions, such as motor imagery, spatial navigation (Sato et al, 2006), memory retrieval (Lundstrom et al, 2005), and also selfperception and internal mentation (Gusnard et al, 2001). On the lateral parietal cortex, IPL which is a heteromodal association area, also has a crucial role in integrating incoming sensory information (Lynch, 1980) and in executive functions, specifically WM, sustained attention and dual task performance (Torrey, 2007). Increased connectivity of parietal regions within the DMN may be compensatory for deficits in other brain regions within the DMN, for example, pCC.

The DMN anti-correlated network comprised a set of brain regions that are recruited during WM tasks (Callicott et al, 1999). Patients with schizophrenia had increased connectivity in DLPFC which reflects the well-known prefronto-cortical dysfunction in schizophrenia (Weinberger et al, 1986). Consistent with these prefrontal effects during working memory, after 8 weeks of treatment with olanzapine, patients with schizophrenia had greater connectivity in vmPFC. Interestingly, connectivity in vmPFC correlated significantly with behavioral accuracy in patients at the first time point only and not in controls. On the other hand, DLPFC activation in healthy subjects correlated with WM behavioral performance both at $t_{1}$ and $t_{2}$. Alterations in vmPFC have been reported in patients with schizophrenia and this finding has been associated with abnormal prefronto-cortical function (Meyer-Lindenberg et al, 2001; Pomarol-Clotet et al, 2008). vmPFC is involved in monitoring and integrating emotional and cognitive processing through its projections to limbic regions, orbitofrontal cortex, ventral striatum, amygdale, and hypothalamus (Gusnard et al, 2001). 
This brain region has an important role in decision making as well as in emotion and motivation associated with a cognitive task. Neurons in vmPFC encode the relative current value and the outcome of chosen options during decisions by comparing available options (Boorman et al, 2009; Luk and Wallis, 2009). In addition, vmPFC regulates both the emotional context and motivational milieu during a cognitive task (Groenewegen and Uylings, 2000) so that this region inhibits emotional processing that may interfere with cognitive performance in a 'dynamic interplay' with the regions of DMN anticorrelated network and specifically DLPFC (Longe et al, 2009). In keeping with this, Uddin et al (2009) found that DMN activity in vmPFC 'Granger-causes' the activity in the anticorrelated network and not vice versa. Decreased temporal anticorrelation between the DMN and task-related network may impair task performance either for decreased allocation of attentional resources for the task at hand (Fransson, 2006) or for direct interference of non suppressed DMN ('default mode interference hypothesis' Sonuga-Barke and Castellanos, 2007). Our present data in vmPFC are consistent with this earlier literature. Moreover, they suggest that olanzapine treatment is associated with a more physiological modulation of the relationship between vmPFC connectivity and behavioral accuracy which is found only in patients and only at $t_{1}$. This interpretation is also consistent with the possibility that olanzapine treatment may modulate DMN directly or acting on both anticorrelated networks (Bertolino et al, 2004; Blasi et al, 2009). As a result of this modulation, olanzapine can improve the temporal characteristics of brain response (Schlagenhauf et al, 2008) of these networks so that patients have a faster and deeper hemodynamic response to the task at hand (Figure 4) which may improve WM performances.

Olanzapine may affect DMN connectivity via dopaminergic system either directly on pCC or indirectly via PFC modulation. Evidence from animal studies indicates that olanzapine increases extracellular levels of dopamine in PFC (Gessa et al, 2000; Ichikawa et al, 2001). Dopamine can regulate brain networks by modulating the synchronization among different brain regions and membrane oscillation frequencies (Seamans and Yang, 2004) thus increasing stability of attentional networks, which ultimately results in decreased trial-by-trial variability and increased signal-tonoise ratio (Rolls et al, 2008). Decreased dopamine levels as resulting from carrying val alleles of $\mathrm{COMTval}^{158} \mathrm{met}$ polymorphism have been associated with increased brain deactivations in pCC during a prosaccade task (Ettinger et al, 2008) as well as a decreased sequence learning-related deactivation in mPFC (Argyelan et al, 2008). Thus increased levels of dopamine in the hubs of DMN may affect the connectivity in the whole network. Alternatively, treatment with olanzapine may also indirectly regulate the DMN connectivity via an increase of dopamine levels in the anticorrelated network (Bertolino et al, 2004).

A caveat to our study is the lack of fMRI data during WM at baseline that for clinical reasons could not be acquired. It is extremely difficult to obtain co-operation from patients to undergo complicated research experiments during acute exacerbations requiring hospitalization. We cannot exclude that patients may have already had an improvement at the level of brain physiology and of behavioral performance before 4 weeks of treatment with olanzapine relative to the baseline. Nevertheless, our data acquired within the timeframe of this study suggest that olanzapine modulates not only DMN connectivity, but, more importantly, its relationship with WM performance.

Another limitation of this study is the intrinsic reliability of the fMRI data in longitudinal studies. Manoach et al (2001), have reported a low test-retest reliability of the magnitude and extent of brain activations during a Sternberg Item recognition task in patients with schizophrenia across scan sessions. To control this variance, we used a bootstrapped ICA that is a technique well-known to be able to identify and isolate artifactual and noise components (Calhoun et al, 2001).

Finally, although estimation of DMN may be more optimal during non complex cognitive tasks (Esposito et al, 2006), some studies have reported similar spatial extent of DMN during rest and during variously demanding cognitive tasks (Calhoun et al, 2008; Smith et al, 2009). Moreover, Calhoun et al (2008) found similar results during rest and during an oddball task when comparing results between patients with schizophrenia and normal controls. Furthermore, most of the studies on DMN in patients with schizophrenia have used complex cognitive tasks such as an oddball task (Garrity et al, 2007), Multi-Source Interference Task (Harrison et al, 2007) and 1-back and 2-back (Pomarol-Clotet et al, 2008; Whitfield-Gabrieli et al, 2009). These studies have reported results that are similar to those obtained with rest data. The objective of our study was to investigate potential effects of treatment using a within subject design under the same task load conditions. Therefore, this design has allowed estimation and evaluation of our objective under very similar physiological conditions thus contributing to temper potential limitations associated with assessing DMN during cognition.

In conclusion, our data suggest that treatment with olanzapine can modulate brain connectivity in DMN and in its anticorrelated network, and their task-related responses. Furthermore, our findings shed new light on the functional anatomy underlying the DMN network, suggesting also potential different roles of posterior DMN regions in the pathogenesis of schizophrenia.

\section{ACKNOWLEDGEMENTS}

We are thankful to Riccarda Lomuscio, BA, for help with acquisition of the data, and to all subjects who have participated in the study.

\section{DISCLOSURE}

Dr Bertolino has received consultant fees from Eli-Lilly, Astra-Zeneca, and Janssen. The other authors declare that they do not have any commercial or financial involvements that might present an appearance of a conflict of interest in connection with the submitted article.

\section{REFERENCES}

Argyelan M, Carbon M, Ghilardi MF, Feigin A, Mattis P, Tang C et al (2008). Dopaminergic suppression of brain deactivation responses during sequence learning. J Neurosci 28: 10687-10695. 
Bertolino A, Caforio G, Blasi G, De Candia M, Latorre V, Petruzzella $\mathrm{V}$ et al (2004). Interaction of COMT (Val(108/ 158)Met) genotype and olanzapine treatment on prefrontal cortical function in patients with schizophrenia. Am J Psychiatry 161: 1798-1805.

Blasi G, Popolizio T, Taurisano P, Caforio G, Romano R, Di Giorgio A et al (2009). Changes in prefrontal and amygdala activity during olanzapine treatment in schizophrenia. Psychiatry Res 173: 31-38.

Boorman ED, Behrens TE, Woolrich MW, Rushworth MF (2009). How green is the grass on the other side? Frontopolar cortex and the evidence in favor of alternative courses of action. Neuron 62: 733-743.

Broyd SJ, Demanuele C, Debener S, Helps SK, James CJ, SonugaBarke EJ (2009). Default-mode brain dysfunction in mental disorders: a systematic review. Neurosci Biobehav Rev 33: 279-296.

Buckner RL, Andrews-Hanna JR, Schacter DL (2008). The brain's default network: anatomy, function, and relevance to disease. Ann NY Acad Sci 1124: 1-38.

Buckwalter JA, Parvizi J, Morecraft RJ, van Hoesen GW (2008). Thalamic projections to the posteromedial cortex in the macaque. J Comp Neurol 507: 1709-1733.

Calhoun VD, Adali T, Pearlson GD, Pekar JJ (2001). A method for making group inferences from functional MRI data using independent component analysis. Hum Brain Mapp 14: 140-151.

Calhoun VD, Kiehl KA, Pearlson GD (2008). Modulation of temporally coherent brain networks estimated using ICA at rest and during cognitive tasks. Hum Brain Mapp 29: 828-838.

Callicott JH, Mattay VS, Bertolino A, Finn K, Coppola R, Frank JA et al (1999). Physiological characteristics of capacity constraints in working memory as revealed by functional MRI. Cereb Cortex 9: $20-26$

Castellanos FX, Margulies DS, Kelly C, Uddin LQ, Ghaffari M, Kirsch A et al (2008). Cingulate-precuneus interactions: a new locus of dysfunction in adult attention-deficit/hyperactivity disorder. Biol Psychiatry 63: 332-337.

Cavada C, Goldman-Rakic PS (1989). Posterior parietal cortex in rhesus monkey: I. Parcellation of areas based on distinctive limbic and sensory corticocortical connections. J Comp Neurol 287: 393-421.

Eichele T, Debener S, Calhoun VD, Specht K, Engel AK, Hugdahl K et al (2008). Prediction of human errors by maladaptive changes in event-related brain networks. Proc Natl Acad Sci USA 105: 6173-6178.

Esposito F, Bertolino A, Scarabino T, Latorre V, Blasi G, Popolizio $\mathrm{T}$ et al (2006). Independent component model of the defaultmode brain function: assessing the impact of active thinking. Brain Res Bull 70: 263-269.

Ettinger U, Kumari V, Collier DA, Powell J, Luzi S, Michel TM et al (2008). Catechol-O-methyltransferase (COMT) val158met genotype is associated with BOLD response as a function of task characteristic. Neuropsychopharmacology 33: 3046-3057.

First MB, Gibbon M, Spitzer RL, Williams JBW (1996). User's Guide for the Structured Clinical Interview for DSM IV Axis I Disorders (SCID-I, Research Version). Biometric Research Department: New York.

Fox MD, Snyder AZ, Vincent JL, Corbetta M, Van Essen DC, Raichle ME (2005). The human brain is intrinsically organized into dynamic, anticorrelated functional networks. Proc Natl Acad Sci USA 102: 9673-9678.

Fox MD, Zhang D, Snyder AZ, Raichle ME (2009). The global signal and observed anticorrelated resting state brain networks. J Neurophysiol 101: 3270-3283.

Fransson P (2006). How default is the default mode of brain function? Further evidence from intrinsic BOLD signal fluctuations. Neuropsychologia 44: 2836-2845.
Friston KJ, Frith CD (1995). Schizophrenia: a disconnection syndrome? Clin Neurosci 3: 89-97.

Garrity AG, Pearlson GD, McKiernan K, Lloyd D, Kiehl KA, Calhoun VD (2007). Aberrant 'default mode' functional connectivity in schizophrenia. Am J Psychiatry 164: 450-457.

Genovese CR, Lazar NA, Nichols T (2002). Thresholding of statistical maps in functional neuroimaging using the false discovery rate. Neuroimage 15: 870-878.

Gessa GL, Devoto P, Diana M, Flore G, Melis M, Pistis M (2000). Dissociation of haloperidol, clozapine, and olanzapine effects on electrical activity of mesocortical dopamine neurons and dopamine release in the prefrontal cortex. Neuropsychopharmacology 22: 642-649.

Goldberg TE, Goldman RS, Burdick KE, Malhotra AK, Lencz T, Patel RC et al (2007). Cognitive improvement after treatment with second-generation antipsychotic medications in firstepisode schizophrenia: is it a practice effect? Arch Gen Psychiatry 64: 1115-1122.

Greicius MD, Krasnow B, Reiss AL, Menon V (2003). Functional connectivity in the resting brain: a network analysis of the default mode hypothesis. Proc Natl Acad Sci USA 100: 253-258.

Greicius MD, Srivastava G, Reiss AL, Menon V (2004). Defaultmode network activity distinguishes Alzheimer's disease from healthy aging: evidence from functional MRI. Proc Natl Acad Sci USA 101: 4637-4642.

Greicius MD, Supekar K, Menon V, Dougherty RF (2009). Restingstate functional connectivity reflects structural connectivity in the default mode network. Cereb Cortex 19: 72-78.

Groenewegen HJ, Uylings HB (2000). The prefrontal cortex and the integration of sensory, limbic and autonomic information. Prog Brain Res 126: 3-28.

Gusnard DA, Akbudak E, Shulman GL, Raichle ME (2001). Medial prefrontal cortex and self-referential mental activity: relation to a default mode of brain function. Proc Natl Acad Sci USA 98: 4259-4264.

Hampson M, Driesen NR, Skudlarski P, Gore JC, Constable RT (2006). Brain connectivity related to working memory performance. J Neurosci 26: 13338-13343.

Harrison BJ, Yucel M, Pujol J, Pantelis C (2007). Task-induced deactivation of midline cortical regions in schizophrenia assessed with fMRI. Schizophr Res 91: 82-86.

Himberg J, Hyvarinen A, Esposito F (2004). Validating the independent components of neuroimaging time series via clustering and visualization. Neuroimage 22: 1214-1222.

Hollingshead A, Redlich F (1958). Social Class and Mental Illness. Wiley: New York.

Ichikawa J, Ishii $\mathrm{H}$, Bonaccorso S, Fowler WL, O'Laughlin IA, Meltzer HY (2001). 5-HT(2A) and $\mathrm{D}(2)$ receptor blockade increases cortical DA release via 5-HT(1A) receptor activation: a possible mechanism of atypical antipsychotic-induced cortical dopamine release. J Neurochem 76: 1521-1531.

Keefe RS, Bilder RM, Davis SM, Harvey PD, Palmer BW, Gold JM et al (2007). Neurocognitive effects of antipsychotic medications in patients with chronic schizophrenia in the CATIE Trial. Arch Gen Psychiatry 64: 633-647.

Kim DI, Manoach DS, Mathalon DH, Turner JA, Mannell M, Brown GG et al (2009). Dysregulation of working memory and defaultmode networks in schizophrenia using independent component analysis, an fBIRN and MCIC study. Hum Brain Mapp 30: 3795-3811.

Li YO, Adali T, Calhoun VD (2007). Estimating the number of independent components for functional magnetic resonance imaging data. Hum Brain Mapp 28: 1251-1266.

Liang M, Zhou Y, Jiang T, Liu Z, Tian L, Liu H et al (2006). Widespread functional disconnectivity in schizophrenia with resting-state functional magnetic resonance imaging. Neuroreport 17: 209-213. 
Longe O, Senior C, Rippon G (2009). The lateral and ventromedial prefrontal cortex work as a dynamic integrated system: evidence from FMRI connectivity analysis. J Cogn Neurosci 21: 141-154.

Luk CH, Wallis JD (2009). Dynamic encoding of responses and outcomes by neurons in medial prefrontal cortex. J Neurosci 29: 7526-7539.

Lundstrom BN, Ingvar M, Petersson KM (2005). The role of precuneus and left inferior frontal cortex during source memory episodic retrieval. Neuroimage 27: 824-834.

Lynch JC (1980). The functional organization of posterior parietal association cortex. Behav Brain Sci 3: 485-534.

Manoach DS, Halpern EF, Kramer TS, Chang Y, Goff DC, Rauch SL et al (2001). Test-retest reliability of a functional MRI working memory paradigm in normal and schizophrenic subjects. Am J Psychiatry 158: 955-958.

Mason MF, Norton MI, Van Horn JD, Wegner DM, Grafton ST, Macrae CN (2007). Wandering minds: the default network and stimulus-independent thought. Science 315: 393-395.

Mazoyer B, Zago L, Mellet E, Bricogne S, Etard O, Houde O et al (2001). Cortical networks for working memory and executive functions sustain the conscious resting state in man. Brain Res Bull 54: 287-298.

Meyer-Lindenberg A, Poline JB, Kohn PD, Holt JL, Egan MF, Weinberger DR et al (2001). Evidence for abnormal cortical functional connectivity during working memory in schizophrenia. Am J Psychiatry 158: 1809-1817.

Murphy K, Birn RM, Handwerker DA, Jones TB, Bandettini PA (2009). The impact of global signal regression on resting state correlations: are anti-correlated networks introduced? Neuroimage 44: 893-905.

Nagano-Saito A, Liu J, Doyon J, Dagher A (2009). Dopamine modulates default mode network deactivation in elderly individuals during the Tower of London task. Neurosci Lett 458: 1-5.

Oldfield RC (1971). The assessment and analysis of handedness: the Edinburgh inventory. Neuropsychologia 9: 97-113.

Pomarol-Clotet E, Salvador R, Sarro S, Gomar J, Vila F, Martinez A et al (2008). Failure to deactivate in the prefrontal cortex in schizophrenia: dysfunction of the default mode network? Psychol Med 38: 1185-1193.

Purdon SE, Jones BD, Stip E, Labelle A, Addington D, David SR et al (2000). Neuropsychological change in early phase schizophrenia during 12 months of treatment with olanzapine, risperidone, or haloperidol. The Canadian Collaborative Group for research in schizophrenia. Arch Gen Psychiatry 57: 249-258.

Raichle ME, MacLeod AM, Snyder AZ, Powers WJ, Gusnard DA, Shulman GL (2001). A default mode of brain function. Proc Natl Acad Sci USA 98: 676-682.

Rolls ET, Loh M, Deco G, Winterer G (2008). Computational models of schizophrenia and dopamine modulation in the prefrontal cortex. Nat Rev Neurosci 9: 696-709.
Sambataro F, Murty VP, Callicott JH, Tan HY, Das S, Weinberger DR et al (2008). Age-related alterations in default mode network: impact on working memory performance. Neurobiol Aging. doi:10.1016/J.neurobiolaging.2008.05.022. (in press)

Sato N, Sakata H, Tanaka YL, Taira M (2006). Navigationassociated medial parietal neurons in monkeys. Proc Natl Acad Sci USA 103: 17001-17006.

Sohlagenhauf F, Wustenberg T, Schmack K, Dingas M, Wrase J, Koslowski M et al (2008). Switching schizophrania patients from typical neuroleptics to olanzapine: effects on BOLD response during attention and working memory. Eur Neuro 18: 689-699.

Seamans JK, Yang CR (2004). The principal features and mechanisms of dopamine modulation in the prefrontal cortex. Prog Neurobiol 74: 1-58.

Smith SM, Fox PT, Miller KL, Glahn DC, Fox PM, Mackay CE et al (2009). Correspondence of the brain's functional architecture during activation and rest. Proc Natl Acad Sci USA 106: 13040-13045.

Sonuga-Barke EJ, Castellanos FX (2007). Spontaneous attentional fluctuations in impaired states and pathological conditions: a neurobiological hypothesis. Neurosci Biobehav Rev 31: 977-986.

Talairach J, Tournoux P (1988). Co-Planar Stereotactic Atlas of the Human Brain. Georg Thieme Verlag/Thieme Medical Publishers: Stuttgart-New York.

Torrey EF (2007). Schizophrenia and the inferior parietal lobule. Schizophr Res 97: 215-225.

Uddin LQ, Kelly AM, Biswal BB, Xavier Castellanos F, Milham MP (2009). Functional connectivity of default mode network components: correlation, anticorrelation, and causality. Hum Brain Mapp 30: 625-637.

Weinberger DR, Berman KF, Suddath R, Torrey EF (1992). Evidence of dysfunction of a prefrontal-limbic network in schizophrenia: a magnetic resonance imaging and regional cerebral blood flow study of discordant monozygotic twins. Am J Psychiatry 149: 890-897.

Weinberger DR, Berman KF, Zec RF (1986). Physiologic dysfunction of dorsolateral prefrontal cortex in schizophrenia. I Regional cerebral blood flow evidence. Arch Gen Psychiatry 43: $114-124$.

Weissenbacher A, Kasess C, Gerstl F, Lanzenberger R, Moser E, Windischberger C (2009). Correlations and anticorrelations in resting-state functional connectivity MRI: a quantitative comparison of preprocessing strategies. Neuroimage 47: 1408-1416.

Whitfield-Gabrieli S, Thermenos HW, Milanovic S, Tsuang MT, Faraone SV, McCarley RW et al (2009). Hyperactivity and hyperconnectivity of the default network in schizophrenia and in first-degree relatives of persons with schizophrenia. Proc Natl Acad Sci USA 106: 1279-1284.

Zhou Y, Liang M, Tian L, Wang K, Hao Y, Liu H et al (2007). Functional disintegration in paranoid schizophrenia using resting-state fMRI. Schizophr Res 97: 194-205.

Supplementary Information accompanies the paper on the Neuropsychopharmacology website (http://www.nature.com/npp) 